\title{
QUANTITATIVE ULTRASOUND BIOMICROSCOPY FOR THE ANALYSIS OF HEALTHY AND REPAIR CARTILAGE TISSUES
}

\author{
K. Gelse ${ }^{1,2 \#^{*}}$, A. Olk ${ }^{1 \#}$, S. Eichhorn ${ }^{3}$, B. Swoboda ${ }^{2}$, M. Schoene ${ }^{4,5}$, and K. Raum ${ }^{4,5}$ \\ ${ }^{1}$ Dept. of Orthopaedic Trauma Surgery, University Hospital Erlangen, Germany \\ ${ }^{2}$ Dept. of Orthopaedic Rheumatology, University of Erlangen-Nuremberg, Germany \\ ${ }^{3}$ Dept. of Orthopaedic Sports Medicine, Technical University Munich, Germany \\ ${ }^{4}$ Dept. of Orthopaedics, University Halle-Wittenberg, Germany \\ ${ }^{5}$ Julius Wolff Institut and Berlin-Brandenburg School for Regenerative Therapies, Charité-Universitätsmedizin \\ Berlin, Germany \\ ${ }^{\#} \mathrm{~K}$. Gelse and A. Olk contributed equally to this work
}

\begin{abstract}
The increasing spectrum of different cartilage repair strategies requires the introduction of adequate nondestructive methods to analyse their outcome in-vivo, i.e. arthroscopically. The validity of non-destructive quantitative ultrasound biomicroscopy (UBM) was investigated in knee joints of five miniature pigs. After 12 weeks, six 5-mm defects, treated with different cartilage repair approaches, provided tissues with different structural qualities. Healthy articular cartilage from each contralateral unoperated knee joint served as a control. The reflected and backscattered ultrasound signals were processed to estimate the integrated reflection coefficient (IRC) and apparent integrated backscatter (AIB) parameters. The cartilage repair tissues were additionally assessed biomechanically by cyclic indentation, histomorphologically and immunohistochemically. UBM allowed high-resolution visualisation of the structure of the joint surface and subchondral bone plate, as well as determination of the cartilage thickness and demonstrated distinct differences between healthy cartilage and the different repair cartilage tissues with significant higher IRC values and a steeper negative slope of the depth-dependent backscatter amplitude $\mathrm{AIB}_{\text {slope }}$ for healthy cartilage. Multimodal analyses revealed associations between IRC and the indentation stiffness. Furthermore, $\mathrm{AIB}_{\text {slope }}$ and AIB at the cartilage-bone boundary ( $\mathrm{AIB}_{\mathrm{dC}}$ ) were associated with the quality of the repair matrices and the subchondral bone plate, respectively. This ex-vivo pilot study confirms that UBM can provide detailed imaging of articular cartilage and the subchondral bone interface also in repaired cartilage defects, and furthermore, contributes in certain aspects to a basal functional characterization of various forms of cartilage repair tissues. UBM could be further established to be applied arthroscopically in-vivo.
\end{abstract}

Keywords: acoustic microscopy, ultrasound biomicroscopy, UBM, cartilage repair, chondrocyte transplantation, microfracture, tissue engineering, nondestructive tissue analysis.

*Address for correspondence:

Kolja Gelse

Department of Orthopaedic Trauma Surgery

University Hospital Erlangen

Krankenhausstr. 12

D-91054 Erlangen

Germany

E-mail: kolja.gelse@web.de

\section{Introduction}

The spectrum of different cartilage repair approaches has considerably been increased in recent years including bone marrow stimulating techniques or transplantation of autologous chondrocytes (ACT). Although several strategies have proven their clinical efficacy (Peterson et al., 2002; Dorotka et al., 2005; Knutsen et al., 2007; Saris et al., 2008), the superiority of either method has not finally been proven, and it is still a matter of debate, if the financial burden and complexity of autologous cell transplantation is justified (Wasiak et al., 2006). To determine the putative long-term success of cartilage repair approaches, histological examination of the structural quality of the repair tissues is currently considered the gold standard. However, biopsies may interfere with the integrity of the joint surface and, therefore, are often abandoned from clinical studies. Furthermore, small biopsies may not be representative for the whole joint surface or repair tissue and their histological processing is time consuming.

Thus, there is a requirement to establish methods that allow noninvasive assessment of articular cartilage in vivo. The aim of this study was to investigate the potential of quantitative ultrasound biomicroscopy (UBM) as a nondestructive method for the evaluation of healthy cartilage and cartilaginous repair tissue. Ultrasonic signals that are reflected at the cartilage surface and subchondral bone plate can visualize these interfaces. Furthermore, processing of the reflected and backscattered ultrasonic signals allows the estimation of quantitative parameters that contribute to the characterization of the structural organization and function of the cartilage matrix (Cherin et al., 1998). These parameters include the integrated reflection coefficient (IRC) and the depth-dependent apparent integrated backscatter amplitude (AIB). IRC is a quantitative index of the acoustic energy reflected from the joint surface, which is primarily determined by the composition and structural organization of the superficial cartilage layer. AIB represents the level of acoustic energy backscattered from the cartilage internal structure and reflects composition and structural organization of the cartilage extracellular matrix (ECM) (Cherin et al., 1998; Cherin et al., 2001) as well as morphology and distribution of cells embedded in the ECM (Raum et al., 2007).

Recent studies have shown that the IRC and AIB are sensitive parameters to detect changes of articular cartilage that occur in ageing, osteoarthritis or following collagenase treatment (Cherin et al., 1998; Foster et al., 2000; Cherin et al., 2001; Spriet et al., 2005; Saarakkala 
et al., 2006; Kiviranta et al., 2007). However, the validity of these quantitative ultrasound parameters has not been shown for the evaluation and characterization of cartilage repair tissues.

This pilot study investigated the applicability of UBM in the knee joint of miniature pigs, which is an established animal model for cartilage repair studies (Hunziker et al., 2003; Blanke et al., 2009; Jung et al., 2009). The aim was to capture various structural and functional parameters associated to surface morphology, cell morphology and distribution, extracellular matrix composition, and subchondral bone integrity. To yield a broad range of cartilage repair tissue properties and different structural patterns, six established repair strategies based on microfracture or autologous chondrocyte transplantation were applied in the knee joint and compared with healthy articular cartilage. For the interpretation of the ultrasound data, the repair tissues as well as healthy cartilage were also analyzed biomechanically by cyclic indentation tests, and morphologically by histological examination using the ICRS visual assessment scale (Mainil-Varlet et al., 2003).

\section{Materials and Methods}

\section{Isolation and culture of autologous chondrocytes}

Five female adult miniature pigs aged 18 months (Ellegaard, Dalmose, Denmark) with body weights of 35$40 \mathrm{~kg}$ were used in this study.

For isolation of autologous chondrocytes, the pigs were anesthetized by intramuscular injection of $30 \mathrm{mg}$ midazolam (Roche, Mannheim, Germany) and $300 \mathrm{mg}$ ketamine (Pfizer, New York, NY, USA) followed by ventilation with isoflurane (Baxter Diagnostic, McGaw Park, IL, USA) at $2 \mathrm{~L} / \mathrm{min}$. The cells were obtained from the chondral part of the $10^{\text {th }} \mathrm{rib}$, a cell source providing phenotypically stable chondrocytes that are characterized by a high proliferative capacity and transcriptional activity of cartilage-specific genes (Xu et al., 2004; Isogai et al., 2006). The harvested cartilage tissue was minced and treated with $0.2 \%$ trypsin (Gibco Life Technologies, Grand Island, NY, USA) for 20 minutes, followed by exposure to $0.02 \%$ clostridial collagenase dissolved in Dulbecco's modified Eagle's medium (DMEM; Gibco Life Technologies) with $10 \%$ fetal calf serum (FCS; Gibco Life Technologies) for 10 hours at $37^{\circ} \mathrm{C}$. Cell culture was performed at $37^{\circ} \mathrm{C}, 5 \% \mathrm{CO}_{2}$, in DMEM supplemented with $10 \% \mathrm{FCS}$ and penicillin/streptomycin (100 units $/ \mathrm{ml})$. The cells were passaged after reaching confluence and transferred to a type I/III collagen membrane (Chondrogide $\AA$; Geistlich Biomaterials, Wolhusen, Switzerland) 48 hours prior to transplantation at a density of $2 \times 10^{6}$ cells $/ \mathrm{cm}^{2}$.

\section{Surgical procedures}

Three weeks after cell isolation the animals were anesthetized as described above. Unilateral arthrotomies of the left knee joints were performed in all animals by a medial parapatellar incision. Six cartilage defects, three each on both aspects of the retropatellar groove of the femoral trochlea, were created using a biopsy punch with
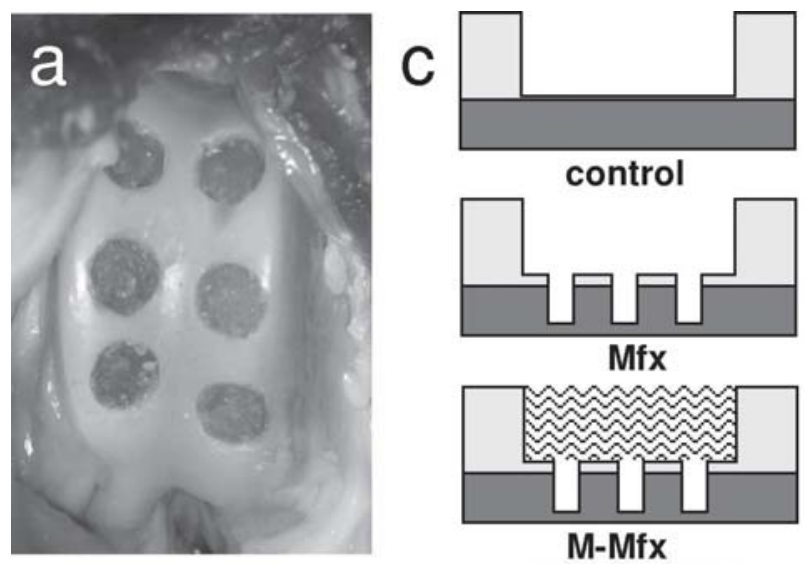

control

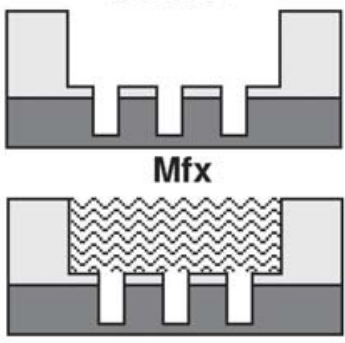

M-Mfx
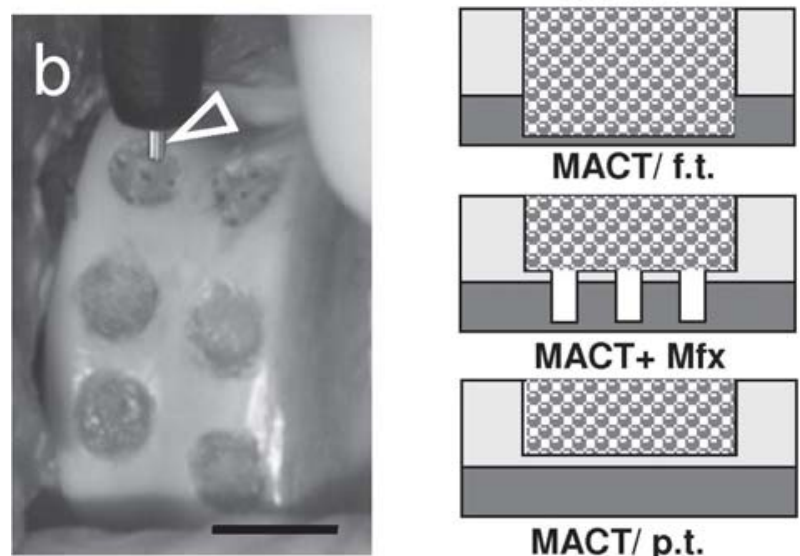

MACT/ f.t.

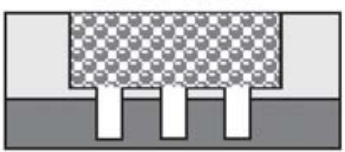

MACT+ Mfx

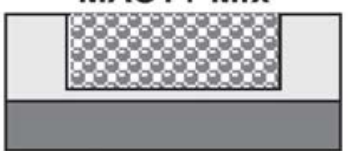

MACT/ p.t.

Fig. 1: Schematic overview of the differentially treated cartilage lesions. Arrangement of the lesions in the femoral trochlea in the knee joint of miniature pigs (a). Intraoperative snapshot of the application of microfractures with a 1-mm thick pick (arrowhead) that exceeded the grip by $3 \mathrm{~mm}$ (b). The defects were treated by the following six different treatment schemes that were arranged in an alternating manner within in the femoral trochlea between the five animals (c): control $=$ untreated control defect with intact subchondral bone plate; $\mathrm{Mfx}=$ microfracture; $\mathrm{M}-\mathrm{Mfx}=$ microfracture covered with Chondrogide ${ }^{\circledR}$ membrane; MACT/ f.t. = matrix-associated autologous chondrocyte transplantation in full-thickness defect; MACT + Mfx $=$ matrix-associated autologous chondrocyte transplantation in microfractured defect; $\mathrm{MACT} /$ p.t. = matrix-associated autologous chondrocyte transplantation in partial-thickness defect. Bar $=1 \mathrm{~cm}$

$5 \mathrm{~mm}$ in diameter. A distance of about $5 \mathrm{~mm}$ of healthy cartilage remained between the defects (Fig. $1 \mathrm{a}, \mathrm{b}$ ). The cartilage was carefully removed using a curette. The subchondral bone plate was initially left intact, which was confirmed by a complete absence of bleeding. Afterwards, 6 different repair approaches were performed in all five animals as follows (Fig. $1 \mathrm{c}$ ):

(1) Untreated cartilage defect with an intact subchondral bone plate (control lesion); (2) five microfractures (each with a diameter of $1 \mathrm{~mm}$ and a depth of $3 \mathrm{~mm}$ ) without further stimulation ("Mfx") (Fig. 1b); (3) five microfractures covered with a naked Chondrogide $\AA$-collagen membrane (Chondrogide $\AA$, Geistlich, Switzlerland)(“M-Mfx"); (4) application of 
a)

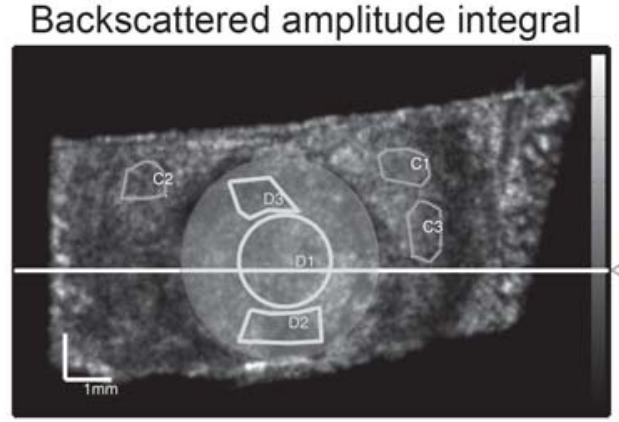

C) Depth dependent backscatter (C1)

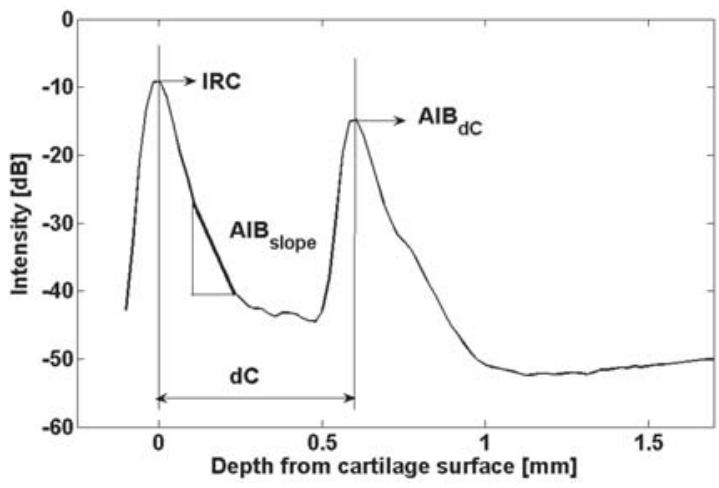

b) B-mode image (cross section)

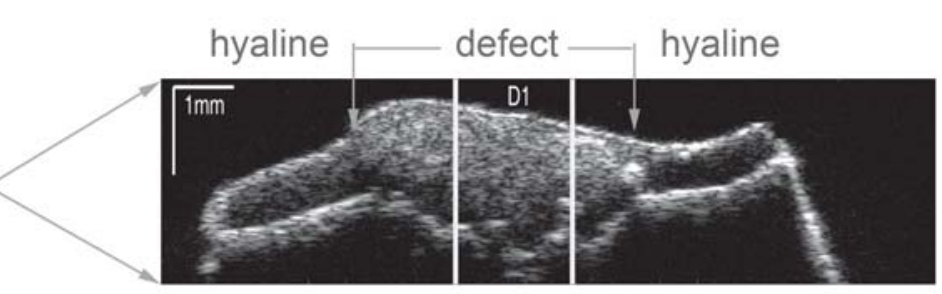

d)

Depth dependent backscatter (D2)

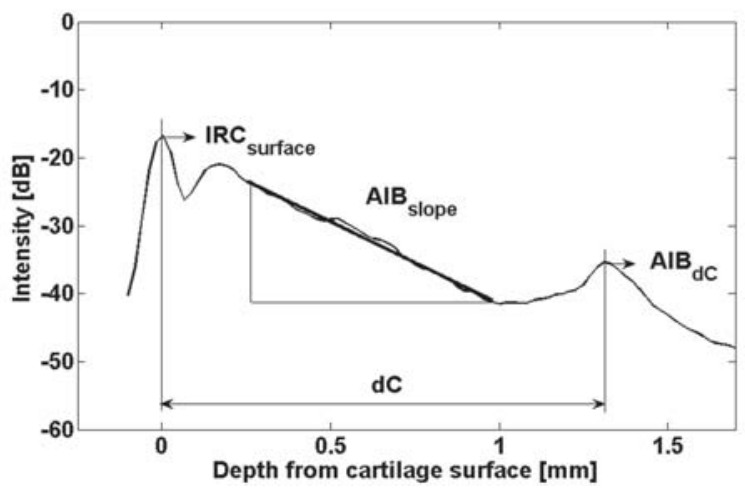

Fig. 2: Example of the ultrasound biomicroscopy analysis of a M-Mfx defect. The backscattered amplitude integral is a top view image of the total backscattered acoustic energy (a). The circular defect region is highlighted in the central part of the image. The B-mode image (b) represents the cross section along the white line in (a). Depthdependent backscatter amplitude curves, averaged within manually selected hyaline cartilage regions (C1-3 as indicated in a) and repair tissue areas (D1-3 as indicated in a) are shown for C1 and D2 in (c) and (d), respectively. Cartilage surface reflection amplitude (first peak), the depth-dependent decrease of the backscatter amplitude and the cartilagebone reflection amplitude (last peak) were used for quantitative evaluation of ultrasound parameters IRC, $\mathrm{AIB}_{\text {slope }}$ and $\mathrm{AIB}_{\mathrm{dC}}$.

collagen membrane (Chondrogide $\left.{ }^{\circledR}\right)$ loaded with autologous chondrocytes (= matrix-associated autologous chondrocyte transplantation, "MACT") within a fullthickness ("f.t.") defect with a completely removed subchondral bone plate ("MACT/ f.t."). Complete removal of the subchondral bone plate was performed using a surgical ball bur; (5) MACT within a cartilage defect with additional five microfractures ("MACT+Mfx"). (6) MACT within a partial-thickness (p.t.) cartilage defect characterized by an intact subchondral bone plate ("MACT/ p.t.").

The size of the Chondrogide $₫$-collagen membrane was fitted to the $5 \mathrm{~mm}$ diameter of the defects. Fixation of the grafts was then achieved by using fibrin glue (Beriplast $\AA$, Aventis Behring, Marburg, Germany). The fibrin glue also served as a sealing of the grafts to prevent dislocation of the membrane-bound chondrocytes. The stability of the grafts was confirmed by repeated flexion of the knee joint with a repositioned patella. Joint capsule and skin were closed with vicryl sutures. Between the five animals, the different treatment approaches were arranged in an alternating manner within the femoral trochlea to avoid any bias by putative local biomechanical differences. Preceding studies on other miniature pigs confirmed no significant spatial differences with respect to the thickness and morphology of articular cartilage and subchondral bone plate within the treatment area of the femoral trochlea.

After surgery, the animals were allowed to move freely in their cages and were sacrificed 12 weeks later by intramuscular injection of $30 \mathrm{mg}$ midazolam and $300 \mathrm{mg}$ ketamine, followed by intravenous bolus-injection of 80 mval $\mathrm{KCl}$ in deep anaesthesia. The knee joints were dissected carefully and the former defect areas of the femoral trochlea were first assessed macroscopically. Osteochondral tissue blocks, which completely contained the respective repair tissues, were cut out of the femur and kept in sterile PBS to prevent drying. Corresponding samples of healthy cartilage were isolated in the same manner from the untreated contralateral right knee joints. The animal study was approved by the appropriate Institutional and Governmental Review Boards (Government of Mittelfranken, Germany; Reference Number 54-2531.31-11/07; Date of Approval 19. Sept. 2007). The governmental regulations for the care and use of laboratory animals have been observed.

\section{High resolution ultrasound biomicroscopy (UBM)}

A custom scanning acoustic microscope SAM200Ex (QBAM, Halle, Germany) was used for the ultrasonic evaluation (Raum et al., 2006; Raum et al., 2007; Leicht et al., 2008; Raum, 2008). A spherically focused lithium niobate transducer $\left(f^{*}=2.66\right.$, NIH Resource Center for 
Ultrasonic Transducer Technology, Los Angeles, CA, USA) provided confocal lateral and axial resolutions of approximately $120 \mu \mathrm{m}$ and $50 \mu \mathrm{m}$, respectively. The specimens were completely immersed in a temperature controlled tank filled with phosphate buffered saline (PBS) at $36^{\circ} \mathrm{C}$. The sample surfaces were placed approximately $0.5 \mathrm{~mm}$ above the focal plane of the transducer. Timeresolved $\mathrm{C}$-scans of the entire defect including the adjacent healthy tissue were performed. For each scanned point the entire pulse echo signal was stored. A scan increment of $20 \mu \mathrm{m}$ was chosen for the acquisition in $\mathrm{x}$ and $\mathrm{y}$ directions. From this three-dimensional data set an overview image (Fig. 2a) and consecutive cross-sectional B-mode images (Fig. 2b) were reconstructed. A 2-mm diameter region in the central part of the defect and up to three areas in the adjacent healthy tissue regions were selected for a depthdependent ultrasound backscatter analysis (Fig. 2c, d) (Raum et al., 2007).

Briefly, a part of the signal was gated using a sliding Hanning window. The positions of the first and last windows were placed $100 \mu \mathrm{m}$ above and $2500 \mu \mathrm{m}$ below the detected cartilage surface reflection, respectively. The gate width was equivalent to the 2 -fold pulse duration (120 $\mathrm{ns})$, which corresponds to a depth range of $\sim 100 \mu \mathrm{m}$ assuming a sound velocity of $1660 \mathrm{~m} / \mathrm{s}$ in cartilage. The overlap between adjacent gate windows was set to $80 \%$. The logarithmic power spectrum was normalized to the reference spectrum obtained from a plane titanium reflector positioned at the same depth, yielding the sound field corrected backscatter spectrum. The apparent integrated backscatter amplitude AIB was obtained by integration in the frequency range between $25 \mathrm{MHz}$ and $45 \mathrm{MHz}$ (Cherin et al., 1998; Cherin et al., 2001).

The depth-dependent AIB amplitude was averaged within manually selected regions of interest (Fig. 2a). The inclination corrected integrated reflection coefficient (IRC) was measured at the cartilage surface. The slope of AIB within the cartilage matrix was estimated by linear regression within a depth-region exhibiting a linear decrease of AIB (Fig. 2c, d). The average cartilage thickness $\mathrm{dC}$ was derived from the distance between the cartilage surface peak to the peak of the backscatter curve at the cartilage-bone boundary. The amplitude $\mathrm{AIB}_{\mathrm{dC}}$ was determined at the cartilage bone interface (Fig. 2c, d).

The proper selection of the evaluation regions, the measurements and the analysis of the data have been performed by a team of two ultrasounds experts (MS and $\mathrm{KR})$.

\section{Cyclic indentation}

Within 24 hours following ultrasound analysis, biomechanical testing of the samples was done as described previously by Maier et al. (Maier et al., 2007). Before and during cyclic indentation testing, the samples were permanently kept in PBS. Cyclic indentation tests were performed as minimally constrained compressionrelaxation tests using a universal testing machine with a spherical indenter tip with a diameter of $5 \mathrm{~mm}$ (Zwicki 1120, Zwick, Ulm, Germany). This indenter was chosen in order to achieve an adequate contact area relative to the defect size. Cyclic indentation was performed in the central part of the respective defect areas corresponding to the site of ultrasound backscatter analysis. Testing was carried out in displacement control. The standardized test cycles comprised the following four phases: preloading of the sample with $0.1 \mathrm{~N}$; dynamic compression with a constant load velocity of $5 \mathrm{~mm} / \mathrm{min}$ until reaching the maximum load of $7 \mathrm{~N}$; static compression of the sample for $60 \mathrm{~s}$ (indenter position was fixed when reaching $7 \mathrm{~N}$ ); relaxation of the sample with a constant load velocity of $1 \mathrm{~mm} / \mathrm{min}$ until a load of $0.15 \mathrm{~N}$. The next cycle started after an interval of $60 \mathrm{~s}$. A total of five cycles were applied. The stiffness was determined from the linear-elastic slope of the loading curve between $2 \mathrm{~N}$ and $5 \mathrm{~N}$.

\section{Histological and immunohistochemical assessment}

After biomechanical testing, the osteochondral specimens were fixed in $4 \%$ paraformaldehyde, followed by decalcification in $0.5 \mathrm{M}$ ethylene diamine tetraacetic acid (EDTA) for 2 months. After standard processing, the samples were embedded in paraffin. Serial transverse 5$\mu \mathrm{m}$ sections were cut through the central part of the defect and were stained with toluidine blue.

Immunohistochemical detection of type I (Col1) and type II (Col2) collagen was performed as described previously in detail (Gelse et al., 2008). Briefly, deparaffinised sections were pretreated with hyaluronidase and pronase, followed by exposure to anti-human type I collagen antibodies (MP Biomedicals, Aurora, OH), or anti-human type II collagen antibodies (MP Biomedicals). After incubation with a biotinylated donkey anti-mouse secondary antibody (Dianova, Hamburg, Germany), a complex of streptavidine and biotinylated alkaline phosphatase was added. The sections were developed with fast red and counterstained with hematoxylin.

The morphological assessment of the different approaches was performed according to the ICRS Visual Histological Assessment Scale (Mainil-Varlet et al., 2003). Representative central sections of the defects were analyzed independently by three experts, and the respective score values were averaged.

\section{Statistical analysis}

For the evaluation of morphological parameters and quantitative ultrasound data non-parametric KruskalWallis tests followed by post-hoc Tukey-Kramer multiple comparison tests were used to determine treatment-specific differences. Cyclic indentation values were assessed by analysis of variance followed by Tukey-Kramer tests. Associations between histological, cyclic indentation and ultrasound values were evaluated by regression analysis. All statistical results were considered significant for $p$ values less than 0.05 .

\section{Results}

\section{Surface characterization of the repair tissue by UBM and histological analysis}

Most of the treated cartilage defects were filled with whitish tissue, but were still distinguishable from adjacent healthy cartilage. The different repair zones did not converge but 
were still strictly separated from each other by an adequate interjacent zone of intact articular cartilage. Therefore, direct crossinfluences in the healing responses between the different repair sites have not been observed.

Ultrasound B-mode images visualized the cartilage surface morphology similar to representative histological sections of each treatment approach (Fig. 3). Lateral integration of repair tissue into adjacent healthy cartilage was tight in all defects except for 2 samples of MACT/p.ttreated lesions with a deep fissure at the interface between graft and adjacent healthy cartilage (Fig. 3f). This fissure was also detectable in the ultrasound B-mode images with an enhanced backscatter signal of this location. Another two failures were observed following MACT/.f.t. and $\mathrm{MACT} /$ p.t., respectively, with delamination of the superficial layer of the collagen membrane. These failures could clearly be visualized by UBM as distinct reflection signals (arrows in Fig. 4 b, c).

\section{Characterization of the tissue matrix by UBM and histological analysis}

In acoustic cross-sectional B-mode images, healthy articular cartilage was typically characterized by a sparse backscatter pattern of its internal matrix (Fig. 3). Instead, fibrous repair tissue or fibrocartilaginous repair tissue displayed more pronounced backscatter signals within the repair matrix that could clearly be distinguished from adjacent healthy cartilage. Increased backscatter signals could particularly be observed in the repair tissues of nontreated lesions (control lesion) (Fig. 3a) and of those covered with a naked collagen membrane (M-Mfx) (Fig. $3 \mathrm{c})$. Both of these repair tissues were typically characterized by a low proteoglycan content and strong staining for type I collagen rather than for the cartilagespecific type II collagen. The tissue generated by Mfx was characterized by a typical bilayer structure: A hyaline-like deep zone staining positive for type II collagen with a minor backscatter pattern could regularly be distinguished from a fibrous superficial zone with a more pronounced backscatter pattern (Fig. 3b).

The treatment by all MACT approaches generated repair matrices with score values significantly superior to those of control defects (Table 1). Histomorphologically, the higher quality of these repair tissues was characterized by a proteoglycan-rich, hyaline-like matrix with distinct metachromatic toluidine blue staining and strong staining for type II collagen, which was reflected by minor ultrasound backscatter signals particularly in deeper zones (Fig. 3 d-f). Increased backscatter signals in superficial zones of some of the MACT-treated lesions may in part be ascribed to remainders of the type I/III collagen membrane, since, in these superficial areas, type I collagen could still immunohistochemically be detected (Fig. 3 d-f; 4 b, c). However, strong staining for type I collagen in superficial layers was predominantly observed in those tissues with a dislocation or delamination of the membrane. Inadequate cell-ingrowth into the dislocated collagen membrane might have interfered with membrane resorption within the follow-up period of 12 weeks. Those areas with a persisting

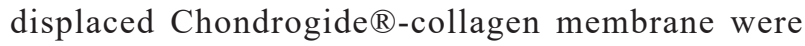

characterized by increased backscatter signals (Fig. 4 b, c).

\section{Reconstitution of the subchondral bone plate and cartilage calcification}

In healthy samples, the second reflection peak (Fig. 2c) can most putatively be ascribed to the tide-mark which is the interface between uncalcified and calcified cartilage and represents a significant acoustic boundary. In the repair tissues, a reconstitution of a physiological tide-mark could histologically not be observed within the follow-up period of 12 weeks. Thus, in these samples, UBM clearly delineated the subchondral bone plate as a hyperechoic reflection band and closely reflected its structure as visualized by histological staining (Fig. 3, 4). Interestingly, irregularities and hypertrophy of the subchondral bone plate could strikingly be observed in non-treated and Mfxtreated defects, both in histological and acoustic images (Fig. 3a,b; Fig. 4a,b). In contrast, such massive hypertrophy of the subchondral bone plate, exceeding its original level, was not observed following MACT-treatment. In partialthickness defects treated by MACT, the initially intact subchondral bone plate was not significantly altered (Fig. 3f) and the transplantation of chondrocytes even prevented ossification and reconstitution of the subchondral bone plate below its original level in defects containing microfractures (Fig. 3e) or in full-thickness defects (Fig. $3 d, 4 b)$.

The clear delineation of the surface and the subchondral bone plate by B-mode images allowed the determination of the average thickness of the uncalcified cartilage repair tissues (Fig. 5). Since MACT prevented ossification of deep layers within full-thickness defects, this treatment resulted in the highest average thickness of the cartilage repair tissues. In contrast, following Mfx-treatment, hypertrophic bone reactions resulted in a low mean thickness of the cartilaginous tissue layer and the irregular structure of the subchondral bone plate was associated with a high standard deviation of the thickness values.

\section{Cyclic indentation}

The stiffness of the freshly isolated native and repair cartilage samples was measured by standardized cyclic indentation protocols. As expected, the stiffness values of native cartilage were significantly higher compared to those of the different repair tissues except for Mfx-treated samples (Fig. 6). Differences between healthy cartilage and repair tissues were significant only for the first indentation cycle with the least precompression. With increasing numbers of cycles, and thus increasing degree of precompression, the differences decreased (data not shown). Differences between the treatment groups by cyclic indentation were statistically not significant. The transplantation of the Chondrogide $\AA$-collagen membrane had no apparent influence on the indentation stiffness of the repair tissues.

\section{Ultrasound backscatter analysis}

Morphological features, e.g. cartilage surface, subchondral bone boundary as well as the border between healthy 


\section{Ultrasound biomicroscopy}

a

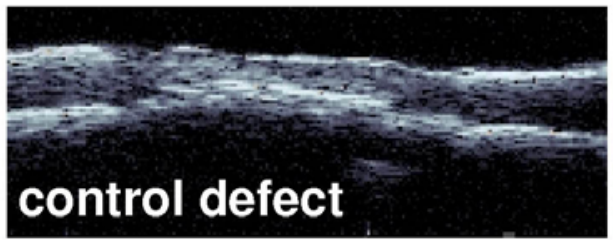

b

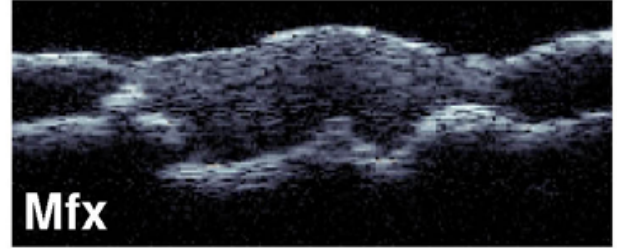

c

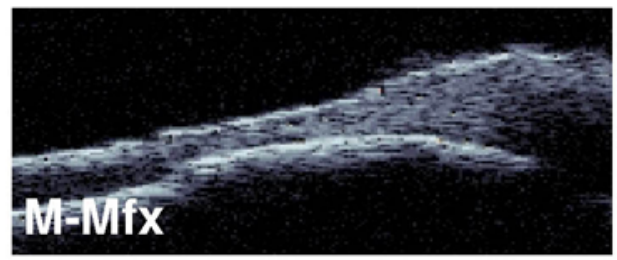

d
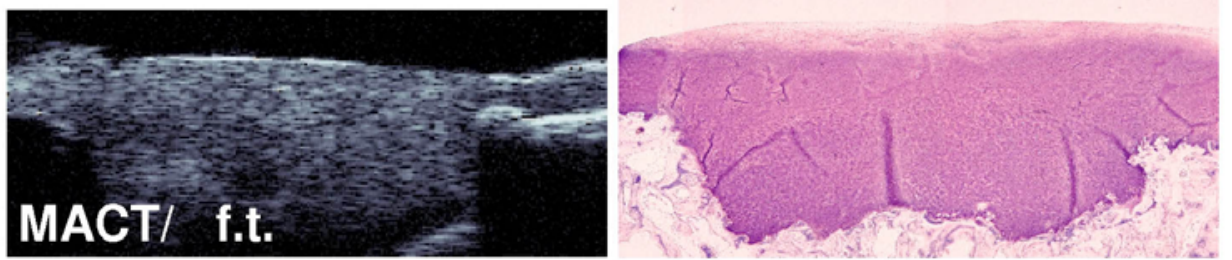

e
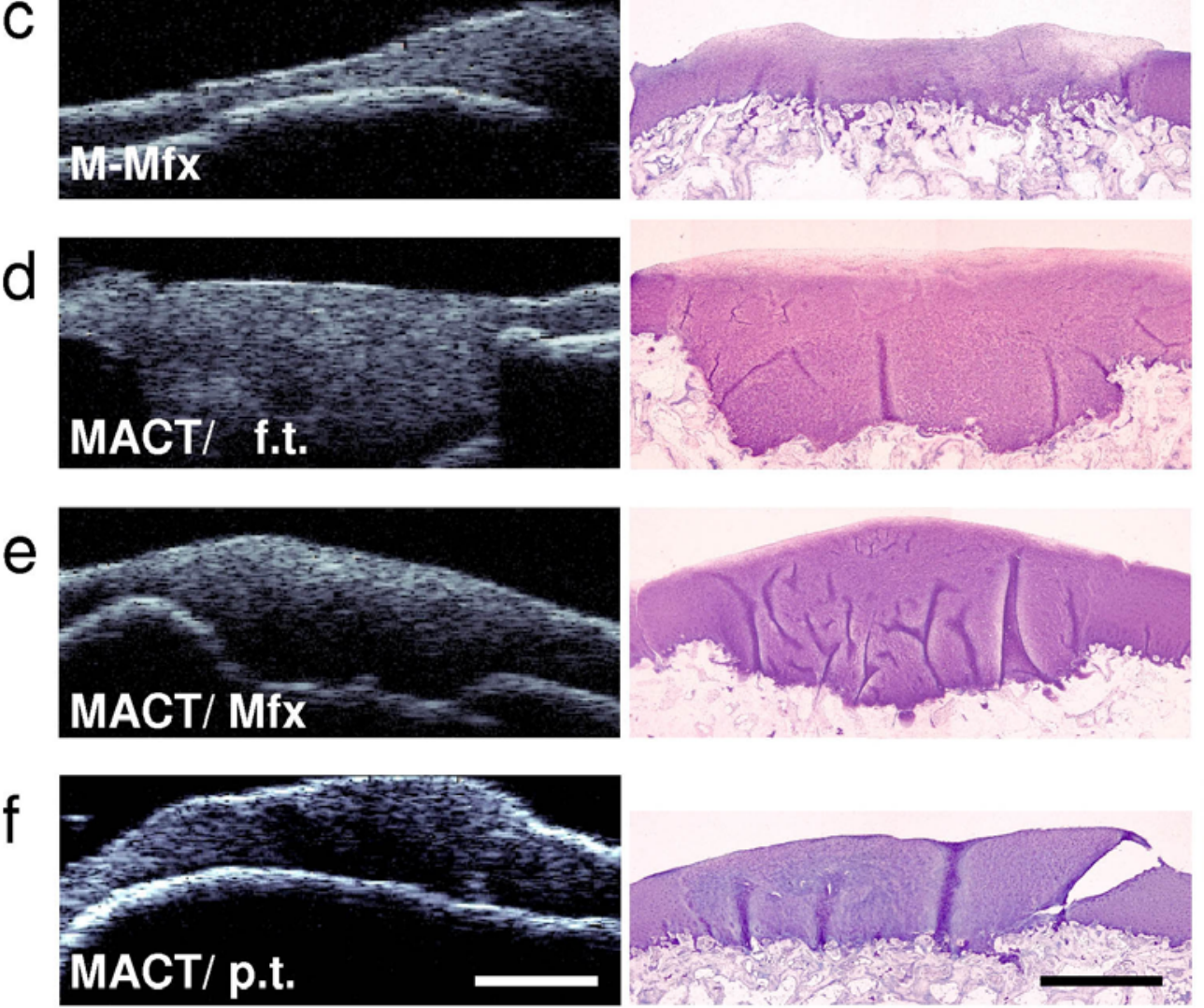
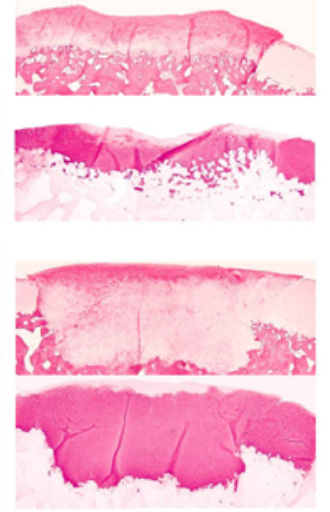

Immunohistochemistry
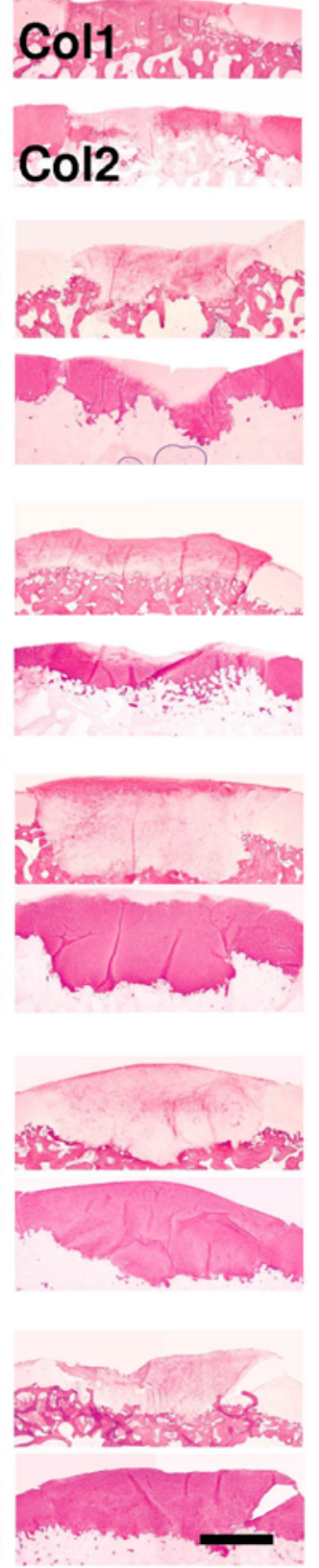

Fig. 3: Representative samples of the different cartilage repair approaches. The resulting repair tissues were assessed histologically by toluidine blue staining and by immunohistochemical staining for type I collagen (Col1) and type II collagen (Col2). Corresponding sections were scanned by ultrasound biomicroscopy. Control defects were mainly filled with fibrocartilaginous tissue (a). Mfx generated hyaline-like repair tissue only in deep zones and fibrocartilaginous tissue in superficial areas (b). The apparent irregular subchondral bone plate was detectable by acoustic microscopy (b). M-Mfx formed fibrocartilaginous tissue with pronounced backscatter signals and predominant staining for type I collagen (c). MACT generated hyaline-like repair tissue that was not replaced by bone in deep zones of full-thickness defects (d) or microfracture cavities (e). The repair tissue following MACT in partial-thickness defects was of high quality but partially failed to merge with adjacent healthy cartilage which was also apparent in the B-mode images (f). Bars $=1 \mathrm{~mm}$. 


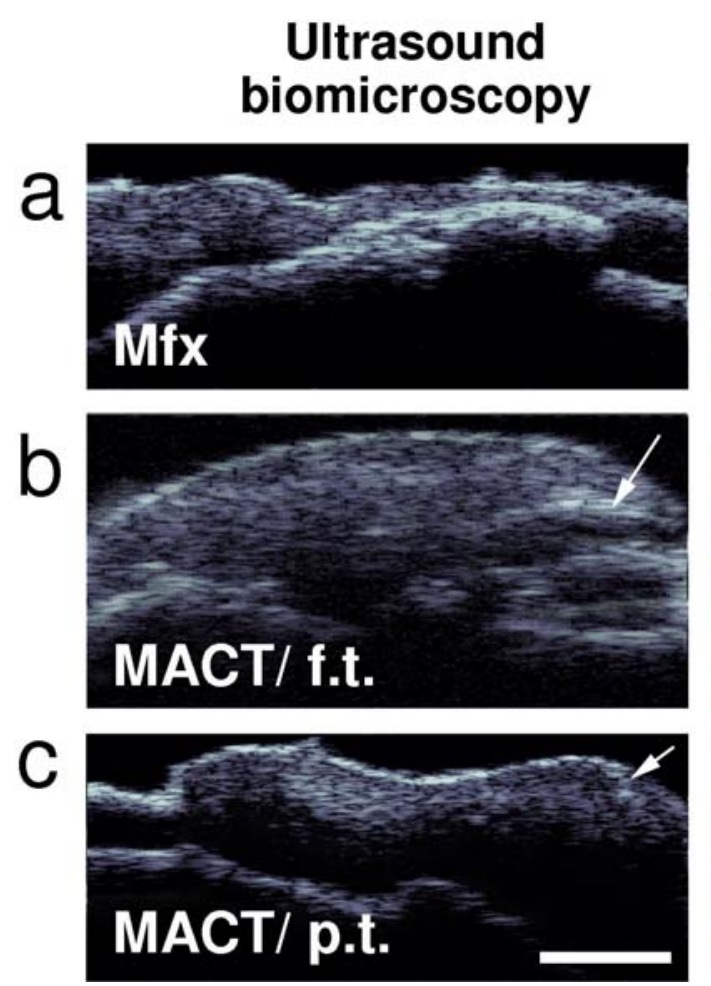

\section{Toluidine blue

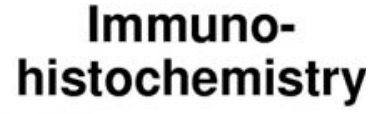

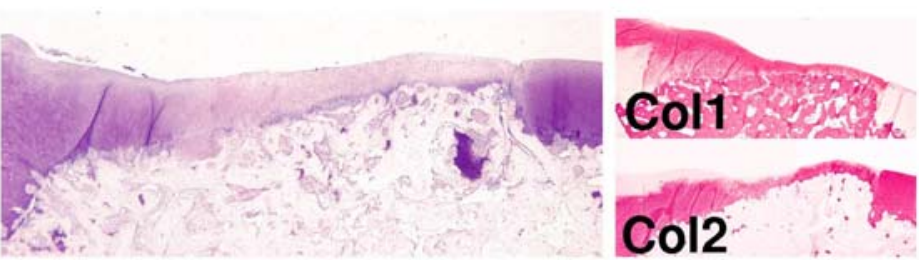
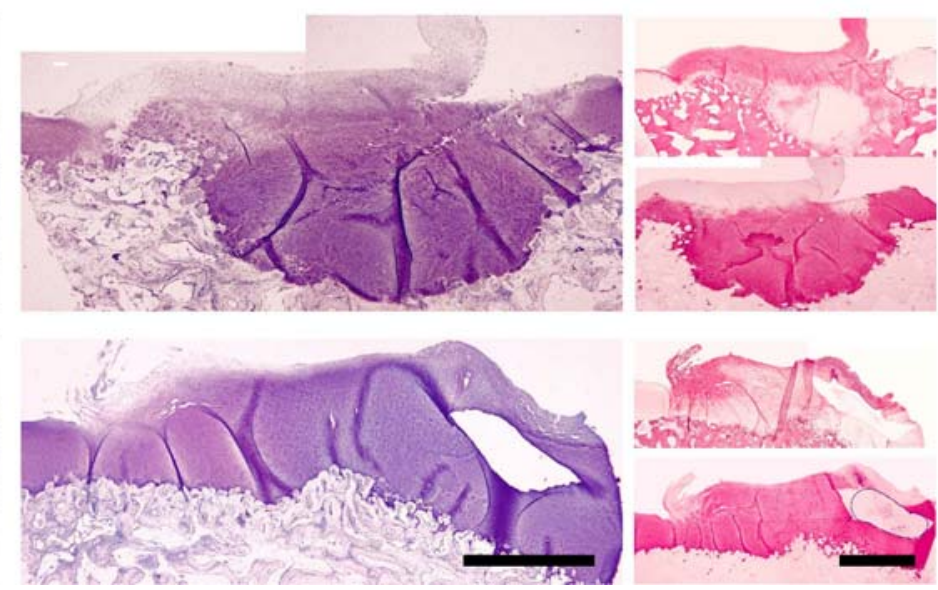

Fig. 4: Detection of failures and worst cases of the different cartilage repair approaches by UBM. Defects treated by microfracturing were confronted with hypertrophy of the subchondral bone plate which could clearly be visualized by acoustic microscopy (a). Acoustic images allowed the detection of delaminations of the collagen membrane that occurred in one MACT-treated full-thickness defect (b) and one MACT-treated partial-thickness defect (c) as indicated by distinct reflection signals (see arrows). Bars $=1 \mathrm{~mm}$.

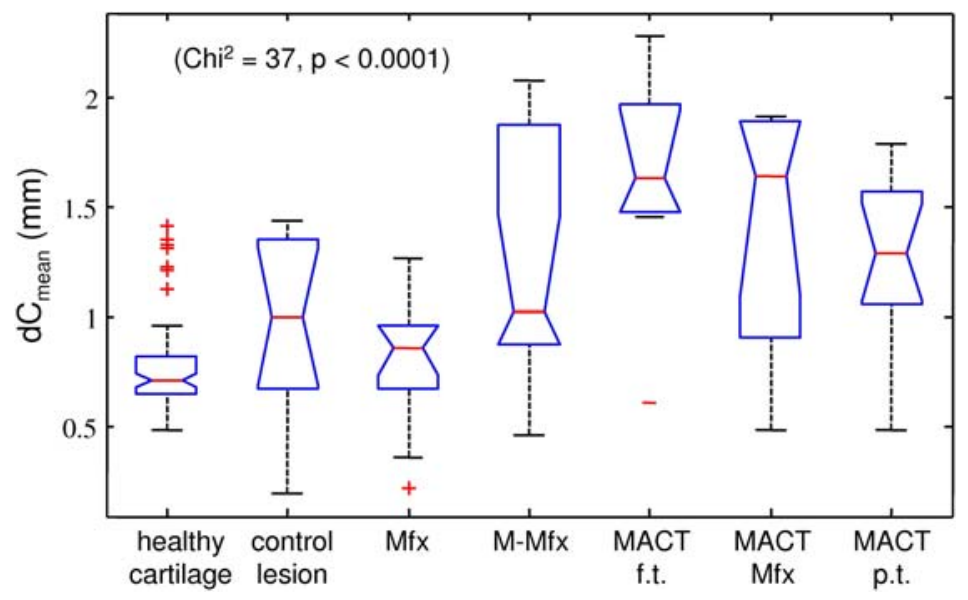

Fig. 5: Thickness of the uncalcified layer of healthy porcine articular cartilage and the different cartilage repair tissues. The thickness values were determined by UBM as the mean distance between the acoustic boundaries at the surface and the uncalcified cartilage- calcified cartilage/bone interface.

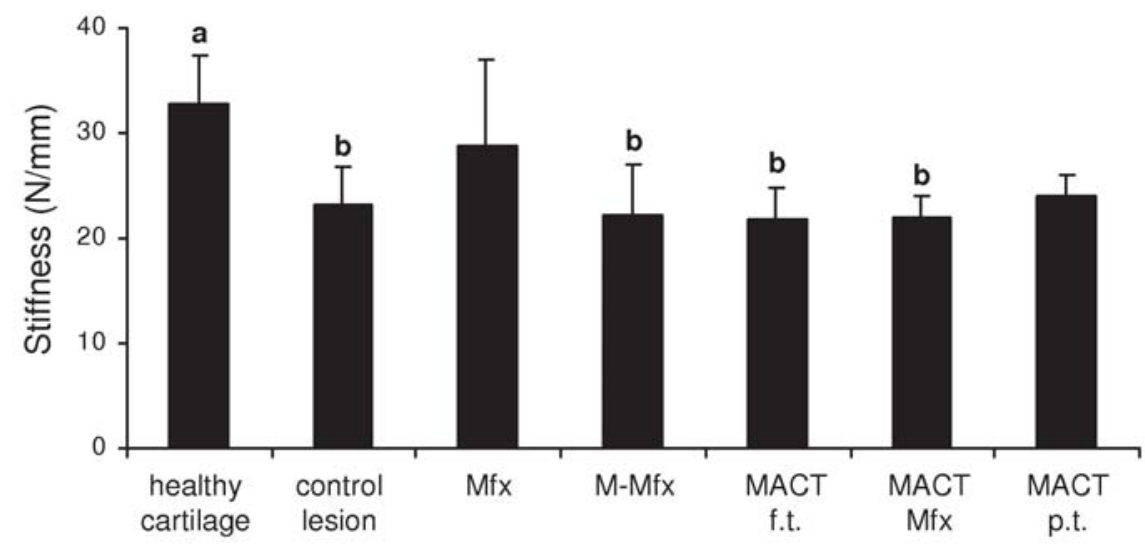

Fig. 6: Biomechanical analysis by cyclic indentation. Results from the first loading cycle are shown. Different superscripts $(a, b)$ denote significantly different values (a vs. $b ; P<0.05)$. 
a)

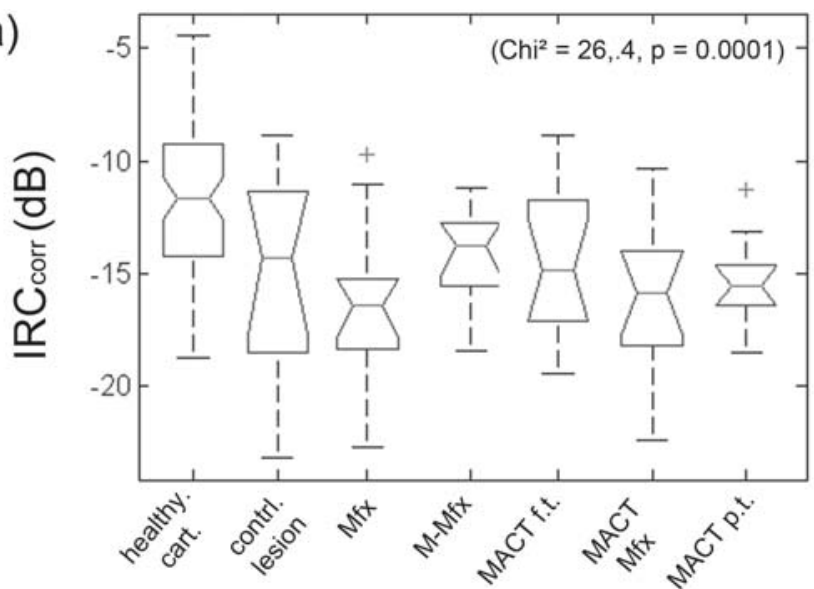

b)

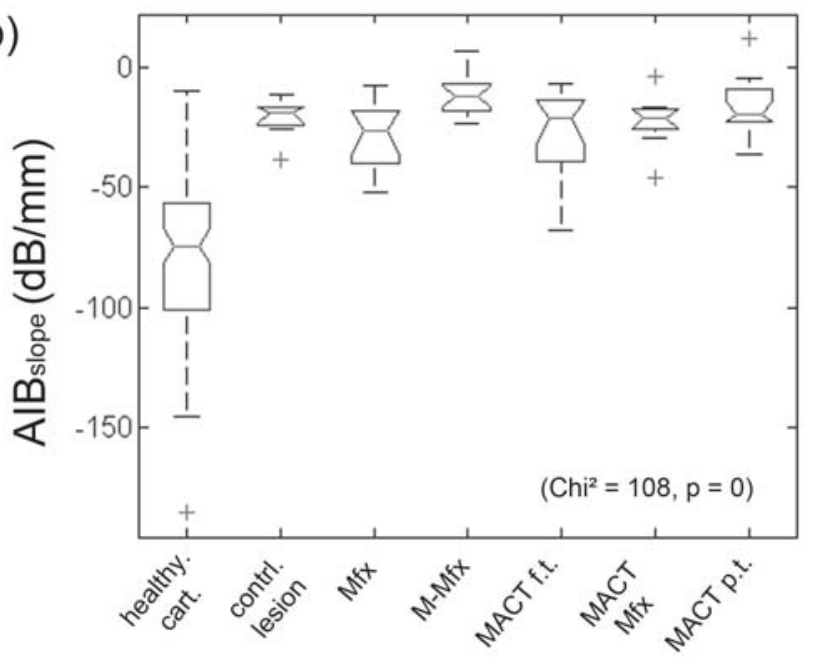

c)

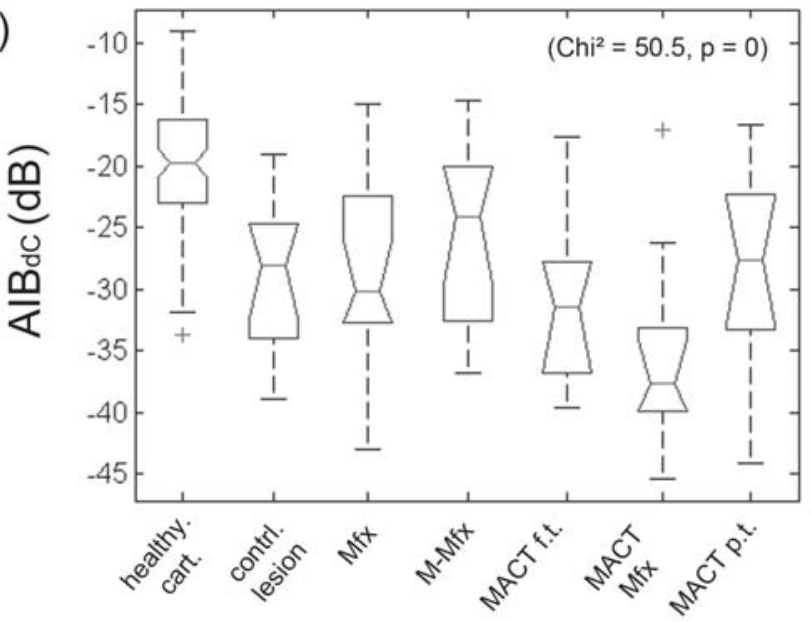

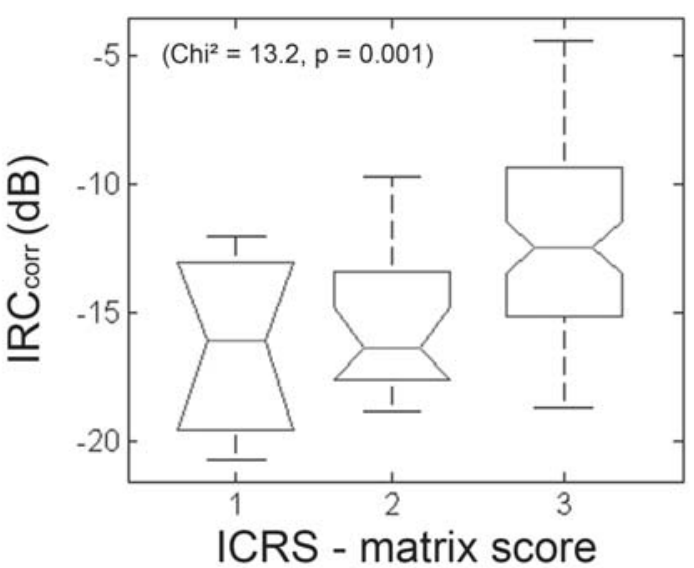
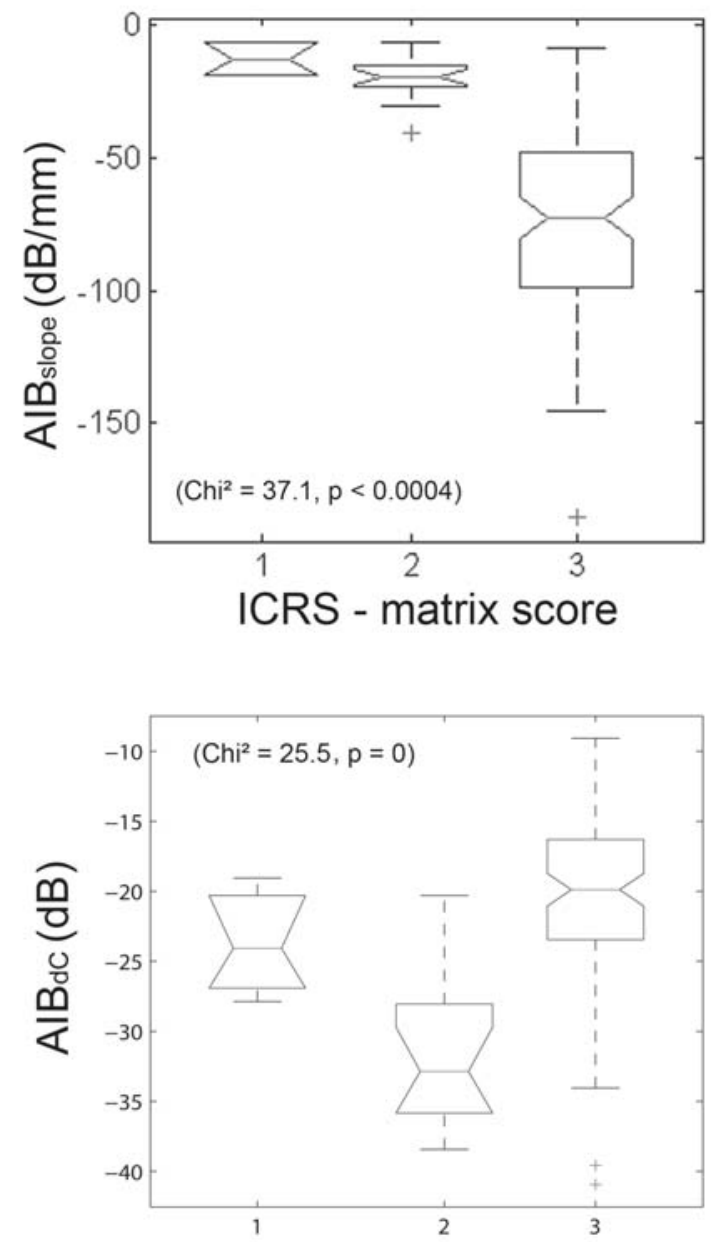

ICRS - subchondral bone score

Fig.7: Quantitative UBM parameters for characterization of healthy cartilage and repair tissues. The inclination corrected integrated reflection coefficient $\operatorname{ICR}_{\text {corr }}$ (a) and the slope of the apparent integrated backscatter $\mathrm{AIB}_{\text {slope }}$ (b) differed significantly between healthy cartilage and repair tissues and were both associated with the histological matrix score. $\mathrm{AIB}_{\mathrm{dC}}$ differed significantly between healthy and repair tissues and was associated with the histological subchondral bone score $(\mathbf{c})$. 


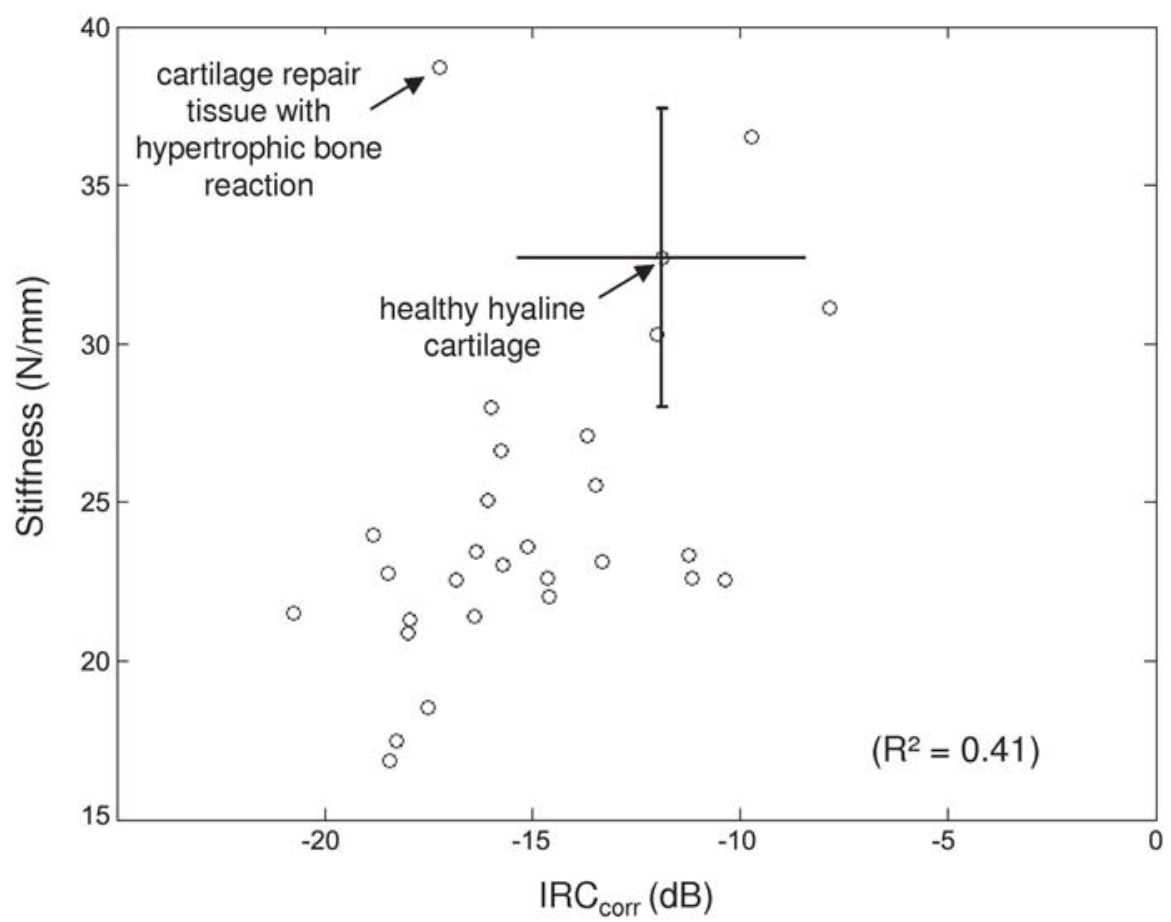

Fig. 8: Stiffness as determined by the first indentation cycle is positively correlated with the inclination corrected integrated reflection coefficient ( $\mathrm{IRC}_{\text {corr }}$ ). Outliers were identified as repair tissue with hypertrophic reactions of the subchondral bone plate. The crossed bars represent mean values and standard deviations of indentation stiffness and $\mathrm{IRC}_{\text {corr }}$ (not site-matched) of all evaluated healthy cartilage regions.

Table 1. Evaluation of the repair tissue according to the ICRS visual histological assessment scale (median, lower and upper quartiles in italic letters). Significant differences are indicated by a $\chi^{2}$ value and different indices ("a","b") denote significantly different values ("a" vs. "b"; $P<0.05$ ).

\begin{tabular}{|c|c|c|c|c|c|c|c|}
\hline \multicolumn{8}{|c|}{ ICRS Score } \\
\hline Treatment & $\mathrm{n}$ & Surface & Matrix & $\begin{array}{c}\text { Cell } \\
\text { distribution }\end{array}$ & $\begin{array}{c}\text { Cell } \\
\text { population } \\
\text { viability }\end{array}$ & $\begin{array}{c}\text { Subchondral } \\
\text { bone }\end{array}$ & $\begin{array}{c}\text { Cartilage } \\
\text { mineralization } \\
\text { (calcified } \\
\text { tissue) }\end{array}$ \\
\hline healthy cart. & 5 & 3 & 3 & 3 & 3 & 3 & 3 \\
\hline control lesion & 5 & $\begin{array}{c}1.00 \\
0.00-1.25\end{array}$ & $\begin{array}{c}1.25^{\mathrm{a}} \\
1.00-1.31\end{array}$ & $\begin{array}{c}0.00 \\
0.00-0.06\end{array}$ & 3.00 & $\begin{array}{c}2.00 \\
1.88-2.25\end{array}$ & $\begin{array}{c}2.00 \\
0.75-2.25\end{array}$ \\
\hline $\mathrm{Mfx}$ & 5 & $\begin{array}{c}1.00 \\
0.00-1.00\end{array}$ & $\begin{array}{c}1.75 \\
1.50-2.00\end{array}$ & $\begin{array}{c}0.00 \\
0.00-0.25\end{array}$ & 3.00 & $\begin{array}{c}2.00 \\
1.88-2.00\end{array}$ & $\begin{array}{c}1.00 \\
0.75-1.63\end{array}$ \\
\hline M-Mfx & 5 & $\begin{array}{c}3.00 \\
2.50-3.00\end{array}$ & $\begin{array}{c}2.00 \\
1.38-2.00\end{array}$ & $\begin{array}{c}0.00 \\
0.00-0.13\end{array}$ & 3.00 & $\begin{array}{c}2.00 \\
1.88-2.13\end{array}$ & $\begin{array}{c}3.00 \\
1.00-3.00\end{array}$ \\
\hline MACT/f.t. & 5 & $\begin{array}{c}3.00 \\
2.50-3.00\end{array}$ & $\begin{array}{c}2.00^{\mathrm{D}} \\
2.00-2.13\end{array}$ & $\begin{array}{c}0.00 \\
0.00-1.00\end{array}$ & 3.00 & $\begin{array}{c}1.50^{\mathrm{D}} \\
1.50-1.63\end{array}$ & $\begin{array}{c}3.00 \\
2.00-3.00\end{array}$ \\
\hline MACT/MfX & 5 & $\begin{array}{c}3.00 \\
0.75-3.00\end{array}$ & $\begin{array}{c}2.00^{\mathrm{b}} \\
2.00-2.63\end{array}$ & $\begin{array}{c}0.00 \\
0.00-1.00\end{array}$ & 3.00 & $\begin{array}{c}1.50^{\mathrm{b}} \\
1.00-1.63\end{array}$ & $\begin{array}{c}3.00 \\
2.75-3.00\end{array}$ \\
\hline MACT/p.t. & 5 & $\begin{array}{c}3.00 \\
2.50-3.00\end{array}$ & $\begin{array}{c}2.00^{b} \\
2.00-3.00\end{array}$ & $\begin{array}{c}1.00 \\
0.75-1.25\end{array}$ & 3.00 & $\begin{array}{c}2.50^{\mathrm{a}} \\
2.38-3.00\end{array}$ & $\begin{array}{c}3.00 \\
2.75-3.00\end{array}$ \\
\hline Chi $^{2}$ & & 14.9 & 18.9 & 8.0 & n.s. & 16.2 & 13.1 \\
\hline
\end{tabular}


Table 2. US parameters with significant associations to ICRS score parameters (Kruskal-Wallis test; $\chi^{2}$ and $p$ values).

\begin{tabular}{lcccc}
\hline & Matrix & Surface & Subchondral Bone & $\begin{array}{c}\text { Cartilage } \\
\text { Mineralisation }\end{array}$ \\
\hline $\mathrm{dC}_{\text {mean }}$ & $14.4(0.0007)$ & & $14.2(0.0002)$ & $13.1(0.005)$ \\
$\mathrm{IRC}_{\text {corr }}$ & $13.2(0.002)$ & & $11.7(0.003)$ & \\
$\mathrm{AlB}_{\text {slope }}$ & $37.1(<0.0001)$ & $12.8(0.005)$ & $36.3(<0.0001)$ & $16.7(0.0002)$ \\
$\mathrm{AlB}_{\mathrm{dc}}$ & $26.3(<0.0001)$ & $12.3(0.006)$ & $25.5(<0.0001)$ & $12.0(0.007)$ \\
\hline
\end{tabular}

cartilage and repair cartilage tissue could easily be distinguished in the acoustic B-mode images (Figs. 2, 3, 4). The quantitative reflection and backscatter parameters contributed to a further characterization of the structure and functional properties of the cartilage matrix.

The inclination corrected IRC of healthy hyaline cartilage was significantly higher than the average IRC values of the different repair tissues $\left(\chi^{2}=26.4\right)$ (Fig. 7a). The differences of the IRC values between the single treatment groups were not significant, but varied significantly between ICRS matrix score values "1" and “3” (Fig. 7a). A significant positive correlation was found between IRC values in the repair tissues and the corresponding indentation stiffness values determined by the first indentation cycle $\left(\mathrm{R}^{2}=0.33\right)$. Outliers from this correlation were identified as repair tissue characterized by significant hypertrophic subchondral bone reactions (Fig. 8). However, no correlation was found for indentation cycles 2 to 5 with increased precompression.

The depth-dependent apparent integrated backscatter (AIB) provided quantitative information of the internal cartilaginous matrix and revealed significant differences between healthy cartilage and repair tissues (Fig. 7b). Hyaline cartilage exhibited a significantly steeper negative slope of AIB compared to all repair tissues $\left(\chi^{2}=108\right)$ (Figs. $2,7 \mathrm{~b})$. Variations of $\mathrm{AIB}_{\text {slope }}$ were also associated with changes of all ICRS scores (Fig. 7 b, Table 2).

The amplitude of AIB at the subchondral bone boundary $A I B_{d C}$ was significantly higher in regions of healthy cartilage than in repair tissues $\left(\chi^{2}=50.5\right)$, except for M-Mfx treated samples (Fig. 7c). Moreover, $\mathrm{AIB}_{\mathrm{dC}}$ were associated with changes of the ICRS scores, in particular those describing the reconstitution of the tissue extracellular matrix and the subchondral bone plate (Fig. 7c, Table 2). Irregularities of subchondral bone structures following Mfx with hyper- and hypotrophic osseous reactions were reflected by a high standard deviation of $\mathrm{AIB}_{\mathrm{dC}}$.

\section{Discussion}

UBM proved to be a valuable non-destructive methodology that allows both morphological and functional characterization of cartilage and other biological tissues (Toyras et al., 1999; Cherin et al., 2001; Nieminen et al.,
2002; Pellaumail et al., 2002; Laasanen et al., 2003; Hattori et al., 2004; Laasanen et al., 2006; Saarakkala et al., 2006; Kiviranta et al., 2007; Raum et al., 2007; Leicht et al., 2008). This study focused on quantitative ultrasound biomicroscopy for the evaluation of cartilage repair tissue. Different cartilage repair approaches were included in this work to provide a divergent spectrum of repair tissues with different matrix qualities, surface characteristics and subchondral bone structures. Considering the limited number of animals and the rather short follow-up period, it was not the scope of this study to determine a general ranking of the different cartilage repair approaches and the apparent superior score values for the "matrix quality" of MACT-treated lesions have to be confirmed in further long-term studies on a larger number of animals. Furthermore, this study did not include explicit assessment of the intra- and interobserver reliability of the histological analysis. In this current pilot study, the different repair tissues rather served as substrates for the evaluation of UBM as a diagnostic tool for the analysis of cartilage repair tissues. For this purpose, UBM analysis was spatially matched to representative histological sections through the central part of each respective defect and to the regions analyzed by mechanical indentation.

For the morphological analysis of the cartilage repair tissues, 40-MHz B-mode analysis proved to be a suitable tool to visualize surface and subchondral bone structures as well as the thickness of the cartilage tissue in close reflection to histological findings. Its high spatial definition did not only depict hypertrophic reactions of the subchondral bone plate following microfracturing, but also sensitively detected delamination of tissue flaps or even small fissures. In fact, such small irregularities represent definite acoustic boundaries that can be visualized by UBM, but may be missed by macroscopic observation or magnetic resonance imaging.

UBM also allowed the assessment of principle conclusions on the three-dimensional structure of the repair matrix. Collagen fibres have been proposed to be the main structural elements within the cartilage extracellular matrix that cause acoustic scattering (Toyras et al., 1999; Pellaumail et al., 2002). This leads to the characteristic depth-dependent backscatter pattern observed for healthy articular cartilage with a high echogeneic superficial layer composed of horizontally aligned collagen fibers, and a nearly echo-free middle and deep zone composed of 
collagen fibres that are primarily oriented perpendicular to the cartilage surface. In contrast, fibrous or fibrocartilaginous repair tissues, with a more irregular structural organization were characterized by strong backscattering throughout the entire thickness of the repair tissue.

The backscatter pattern may also be influenced by remainders of the transplanted Chondrogide ${ }^{\circledR}$ - type I/III collagen membrane. However, in the current work and a previous study, immunohistochemical stainings of the repair tissues for type I collagen indicated that the

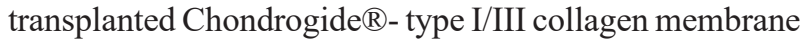
was largely resorbed within a period of 6 to 12 weeks, at least in lesions with an adequate cellular ingrowth and repair response (Gelse et al., 2008). Thus, it can be assumed that, after 12 weeks, the backscatter is primarily reflected by the secondarily remodelled repair tissue that still lacks the characteristic alignment of the collagen fibres present in mature articular cartilage.

The only exclusion could be observed in those lesions with a displaced membrane, which probably interfered with the cellular ingrowth and resorption or remodelling of the transplanted membrane. Those areas were indeed characterized by enhanced backscatter signals. Thus, UBM could also visualize failures of the repair approaches. Notably, UBM visualizes a more native status of the tissue, and histological processing, particularly the fixation process with paraformaldehyde, may lead to some morphological artifacts, e.g. a "coiling" of the displaced membrane (Fig. 4b), or a more pronounced membrane delamination in histological sections (Fig. 4c).

Apart from the B-mode images that are suitable for morphological analysis, further processing of ultrasound signals allowed the estimation of quantitative parameters, which are considered to contribute to the structural and biomechanical characterization of the tissues.

In other studies, the maximum magnitude of the reflected ultrasound signals from the surface has been shown to be associated with the modulus or mechanical stiffness of cartilage tissue or with the histological quality of repair tissue (Laasanen et al., 2003; Hattori et al., 2004; Laasanen et al., 2006; Kuroki et al., 2008). However, it has to be considered that the "intensity" of the reflected ultrasound signals does not represent an intrinsic material property and may also be influenced by several other factors (Saarakkala et al., 2007; Zheng et al., 2008). Further processing of reflected signals allows estimation of the device and operator independent parameter IRC. In this study a significant correlation between IRC and indentation stiffness was apparent for the stiffness values measured with low preload. Similarly, correlations between IRC and the compressive modulus could be also detected for healthy and osteoarthritic cartilage in other studies (Saarakkala et al., 2006; Kiviranta et al., 2007). However, those associations have to be interpreted with care for two reasons:

Firstly, ultrasound reflection at the cartilage surface is primarily determined by the stiffness and integrity (roughness) of the superficial cartilage region, while the stiffness measured with the mechanical cyclic indentation device depends on the bulk stiffness properties over the entire cartilage depth and the subchondral bone plate (Saarakkala et al., 2007). Therefore, a perfect correlation between these parameters could only be expected for homogeneous stiffness properties throughout the entire cartilage depth, which is essentially not the case for repair tissue.

Secondly, it has to be considered that the thickness of the cartilage tissue will affect the mechanical indentation response. Particularly in this animal model, hypertrophic reactions of the subchondral bone plate affected the determination of the stiffness of the overlying softer cartilaginous tissue, which putatively resulted in falsely increased stiffness values for Mfx-treated lesions (Fig. 8). This effect may be particularly relevant in animal models with rather thin articular cartilage and when applying indentation forces with increasing precompression. In our setup, only the first indentation cycle with the least precompression was sensitive enough to detect significant differences for the stiffness of healthy cartilage compared with the repair tissues.

These methodical and biophysical aspects suggest that the validity of the correlation between IRC and the stiffness is limited to the superficial tissue layer. Nevertheless, this restriction may not essentially constrain the diagnostic value of the IRC, since the superficial layer is of great importance for the function of repair tissues. Importantly, shear forces are highest at the surface and degeneration of the articular surface, e.g., fibrillation and fissuring, also begins in these superficial zones (Gelse et al., 2008).

AIB is a quantitative ultrasound parameter that represents an index for the acoustic energy backscattered from the internal matrix. This provides information about the ultrastructural organization of the tissue extracellular matrix. Healthy articular cartilage is typically a highly organized tissue and is characterized by a rapid depthdependent decline in the backscatter amplitude below the superficial layer with horizontally aligned fibrils. In contrast, fibrocartilaginous repair tissue largely lacks this unique zone-dependent microarchitecture and displays a more random alignment of collagen fibrils, which results in a more moderate depth-dependent decline of AIB and backscatter signals throughout all tissue layers. We found that this depth-dependent decline was best represented by the slope of AIB. We described for the first time this parameter as a suitable index for the characterization of cartilage repair tissues.

Statistical analyses revealed a distinct association between the $\mathrm{AIB}_{\text {slope }}$ and the matrix quality of the repair tissues. This reflects the results of former studies comparing healthy cartilage and osteoarthritic or enzymatically degraded cartilage, which showed correlations between AIB and the degree of cartilage degradation (Cherin et al., 2001; Saarakkala et al., 2006). However, alterations in the AIB values may primarily be ascribed to unphysiological alignment or disruption of the collagen network rather than a to lack or depletion of proteoglycans (Cherin et al., 2001). For example, AIB was not influenced by an enzymatically-induced depletion of proteoglycans in a rat model (Pellaumail et al., 2002). 
Within the ICRS scoring system for repair tissues, the parameter "matrix quality" primarily implies the proteoglycan content as a crucial element for its categorization. Since $\mathrm{AIB}_{\text {slope }}$ may not directly reflect the proteoglycan content, its association with the "matrix quality" score seems to be an indirect effect. Nevertheless, $\mathrm{AIB}_{\text {slope }}$ represents a valuable, quantitative index for the extracellular matrix structure of cartilage repair tissues.

The analysis of subchondral bone structures revealed the most striking differences between the different cartilage repair approaches. Hypertrophic and irregular osseous structures following Mfx were found in contrast to a failed or inadequate reconstitution following MACT in fullthickness lesions. The repair tissues within full-thickness or microfractured defects typically failed to reconstitute a tide-mark, at least within the follow-up period of 12 weeks. In healthy articular cartilage, the tide-mark represents a sharp acoustic boundary, since it separates the non-calcified articular cartilage from the underlying calcified cartilage that is characterized by a high degree of mineralization and elastic stiffness (Zizak et al., 2003). Thus, in the case of healthy articular cartilage samples, it can be assumed that the second reflection peak ( $\mathrm{AIB}_{\mathrm{dC}}$-value) (Fig. 2c) is predominantly generated by the sharp acoustic boundary of the tide-mark. In healthy articular cartilage, we could not observe a double-peak of $\mathrm{AIB}_{\mathrm{dC}}$, which indicates that the underlying transition between calcified cartilage and the subchondral bone plate generates only minor backscatter signals. In repair tissue samples lacking a tidemark, $\mathrm{AIB}_{\mathrm{dC}}$ corresponds to the interface between the cartilaginous repair tissue and newly-formed or still existing bone. The irregular structure of the forming bone trabeculae and the rather irregular mineralization of the cartilaginous extracellular matrix with no definite tidemark results in lower values of $\mathrm{AIB}_{\mathrm{dC}}$. In this respect, we defined $\mathrm{AIB}_{\mathrm{dC}}$ as a novel suitable parameter for the characterization of subchondral bone structures. Higher $\mathrm{AIB}_{\mathrm{dC}}$ values reliably reflected a more physiological reconstituted transition from non-calcified cartilage to subchondral bone plate defined by histological examination and small standard deviations indicated a more regular formation of this structure.

Considering these facts, it can also be concluded that UBM determines the thickness of the non-calcified cartilage and does not capture the layer of calcified cartilage. However, the thickness of the calcified cartilage layer in the trochlea of minipigs $(0-0.05 \mathrm{~mm})$ is by far less than $10 \%$ of the thickness of the overlying noncalcified cartilage $(0.6-1 \mathrm{~mm})$ and is, thus, of rather limited diagnostic relevance.

This pilot study demonstrated the potentials but also the limitations of UBM for the characterization of cartilage repair tissues. Center frequency and sound field geometry of our system have been optimized for the investigation of human cartilage, i.e. it provides a sufficient signal-tonoise-ratio combined with a reasonable focus range for a quantitative ultrasound backscatter analysis within the superficial and subsurface cartilage tissue, a sufficient penetration for the detection of the mineralized tidemark or subchondral bone plate even in thicker cartilage regions, and good axial and lateral image resolutions (Raum et al., 2007). While the diagnostic value of B-mode imaging is clearly apparent, the interpretation of the quantitative ultrasound data seems to be more complex and future research is still required to validate potential correlations with conventional structural and biomechanical parameters. To date, valid parameters for the determination of the proteoglycan content by ultrasound analysis have still to be established. For this purpose, the role of sound velocity or frequency dependent attenuation measurements have been discussed in recent studies, however with inconsistent results so far (Pellaumail et al., 2002; Toyras et al., 2003; Kiviranta et al., 2007; Wang et al., 2008).

Due to the rapid expanding field of cartilage repair approaches, non-destructive diagnostic tools for monitoring cartilage repair or cartilage degeneration will increasingly be of interest. Quantitative ultrasound analysis may still have its limitations to discern an unphysiological collagen network from a depletion or lack of proteoglycans (Toyras et al., 1999).

Quantitative MRI is currently the state-of-the-art technology for a noninvasive assessment of articular cartilage. Currently established clinical MRI provides comprehensive information of the entire joint, including cartilage, subchondral bone and synovial membrane (Gold et al., 2009). Recently, a dedicated experimental highresolution MRI with a voxel size of 51 x 51 x $94 \mu \mathrm{m}$ has been reported (Rengle et al., 2009). While multi-echo techniques, e.g. dual excitation in the steady state (DESS), are suitable for morphologic assessment, contrastenhanced imaging, e.g. delayed gadolinium enhanced MRI of cartilage (dGEMRIC), is sensitive to biochemical alterations of the proteoglycan content. Moreover, the organized structure of the collagen network gives rise to a magic angle effect in T2-weighted sequences, which in turn allows an indirect assessment of the orientation of the collagen fibers. Compared to these MRI evaluation techniques, UBM has several complementary potentials: The spatial resolution of UBM is one order of magnitude better than current conventional MRI systems, which allows a separate evaluation of the individual cartilage layers (Raum et al., 2007), and which provides a high sensitivity to surface roughness (Viren et al., 2009). Furthermore, the elastic interaction of the ultrasound wave with the tissue delivers information on alterations of matrix elasticity, collagen orientation, packing density, as well as a depth-dependent information on number, size and shape of cells in the subsuperficial layers (Raum et al., 2007).

Although UBM cannot be applied noninvasively, it represents a cost-effective and mobile diagnostic tool which could ideally be combined with mechanical measurements or MRI, two rather stationary methods, to provide comprehensive biochemical, biomechanical and structural characterization of various cartilage repair tissues. Recently, small ultrasound transducers, that were originally intended for intravascular use, have been shown to be also suited for the analysis of cartilage tissue and even for direct intraarticular diagnostics of articular cartilage (Huang et al., 2009; Viren et al., 2009). Such miniaturized catheter-based devices could be further 
established for arthroscopic use in the operation theatre, which would then allow real-time evaluation and monitoring of cartilage repair tissues in clinical settings.

\section{Acknowledgements}

We thank M. Geßlein and H. Rohrmüller for expert technical assistance. This study was supported by the Interdisciplinary Center of Clinical Research (IZKF) at the University Erlangen (grant A 36), the German Research Foundation (grant Ra 1380/6) and the German Society of Orthopaedics and Orthopaedic Surgery (DGOOC, MSBNet).

\section{References}

Blanke M, Carl HD, Klinger P, Swoboda B, Hennig F, Gelse K (2009) Transplanted chondrocytes inhibit endochondral ossification within cartilage repair tissue. Calcif Tissue Int 85: 421-433.

Cherin E, Saied A, Laugier P, Netter P, Berger G (1998) Evaluation of acoustical parameter sensitivity to agerelated and osteoarthritic changes in articular cartilage using 50-Mhz ultrasound. Ultrasound Med Biol 24: 341 354.

Cherin E, Saied A, Pellaumail B, Loeuille D, Laugier P, Gillet P, Netter P, Berger G (2001) Assessment of rat articular cartilage maturation using $50-\mathrm{Mhz}$ quantitative ultrasonography. Osteoarthritis Cartilage 9: 178-186.

Dorotka R, Bindreiter U, Macfelda K, Windberger U, Nehrer S (2005) Marrow stimulation and chondrocyte transplantation using a collagen matrix for cartilage repair. Osteoarthritis Cartilage 13: 655-664.

Foster FS, Pavlin CJ, Harasiewicz KA, Christopher DA, Turnbull DH (2000) Advances in ultrasound biomicroscopy. Ultrasound Med Biol 26: 1-27.

Gelse K, Muhle C, Franke O, Park J, Jehle M, Durst K, Goken M, Hennig F, Mark KV, Schneider H (2008) Cell-based resurfacing of large cartilage defects: Longterm evaluation of grafts from autologous transgeneactivated periosteal cells in a porcine model of osteoarthritis. Arthritis Rheum 58: 475-488.

Gold GE, Chen CA, Koo S, Hargreaves BA, Bangerter NK (2009) Recent advances in MRI of articular cartilage. AJR Am J Roentgenol 193: 628-638.

Hattori K, Takakura Y, Morita Y, Takenaka M, Uematsu K, Ikeuchi K (2004) Can ultrasound predict histological findings in regenerated cartilage? Rheumatol (Oxford) 43: 302-305.

Huang YP, Zheng YP (2009) Intravascular ultrasound (IVUS): A potential arthroscopic tool for quantitative assessment of articular cartilage. Open Biomed Eng J 3: 13-20.

Hunziker EB, Driesang IM (2003) Functional barrier principle for growth-factor-based articular cartilage repair. Osteoarthritis Cartilage 11: 320-327.

Isogai N, Kusuhara H, Ikada Y, Ohtani H, Jacquet R, Hillyer J, Lowder E, Landis WJ (2006) Comparison of different chondrocytes for use in tissue engineering of cartilage model structures. Tissue Eng 12: 691-703.
Jung M, Breusch S, Daecke W, Gotterbarm T (2009) The effect of defect localization on spontaneous repair of osteochondral defects in a gottingen minipig model: A retrospective analysis of the medial patellar groove versus the medial femoral condyle. Lab Anim 43: 191-197.

Kiviranta P, Toyras J, Nieminen MT, Laasanen MS, Saarakkala S, Nieminen HJ, Nissi MJ, Jurvelin JS (2007) Comparison of novel clinically applicable methodology for sensitive diagnostics of cartilage degeneration. Eur Cell Mater 13: 46-55.

Knutsen G, Drogset JO, Engebretsen L, Grontvedt T, Isaksen V, Ludvigsen TC, Roberts S, Solheim E, Strand T, Johansen O (2007) A randomized trial comparing autologous chondrocyte implantation with microfracture. Findings at five years. J Bone Joint Surg Am 89: 21052112.

Kuroki H, Nakagawa Y, Mori K, Kobayashi M, Yasura K, Okamoto Y, Suzuki T, Nishitani K, Nakamura T (2008) Ultrasound properties of articular cartilage in the tibiofemoral joint in knee osteoarthritis: Relation to clinical assessment (International Cartilage Repair Society grade). Arthritis Res Ther 10: R78.

Laasanen MS, Toyras J, Vasara A, Saarakkala S, Hyttinen MM, Kiviranta I, Jurvelin JS (2006) Quantitative ultrasound imaging of spontaneous repair of porcine cartilage. Osteoarthritis Cartilage 14: 258-263.

Laasanen MS, Toyras J, Vasara AI, Hyttinen MM, Saarakkala S, Hirvonen J, Jurvelin JS, Kiviranta I (2003) Mechano-acoustic diagnosis of cartilage degeneration and repair. J Bone Joint Surg Am 85-A Suppl 2: 78-84.

Leicht S, Raum K (2008) Acoustic impedance changes in cartilage and subchondral bone due to primary arthrosis. Ultrasonics 48: 613-620.

Maier D, Braeun K, Steinhauser E, Ueblacker P, Oberst M, Kreuz PC, Roos N, Martinek V, Imhoff AB (2007) In vitro analysis of an allogenic scaffold for tissue-engineered meniscus replacement. J Orthop Res 25: 1598-1608.

Mainil-Varlet P, Aigner T, Brittberg M, Bullough P, Hollander A, Hunziker E, Kandel R, Nehrer S, Pritzker K, Roberts S, Stauffer E (2003) Histological assessment of cartilage repair: A report by the histology endpoint committee of the International Cartilage Repair Society (ICRS). J Bone Joint Surg Am 85-A Suppl 2: 45-57.

Nieminen HJ, Toyras J, Rieppo J, Nieminen MT, Hirvonen J, Korhonen R, Jurvelin JS (2002) Real-time ultrasound analysis of articular cartilage degradation in vitro. Ultrasound Med Biol 28: 519-525.

Pellaumail B, Watrin A, Loeuille D, Netter P, Berger G, Laugier P, Saied A (2002) Effect of articular cartilage proteoglycan depletion on high frequency ultrasound backscatter. Osteoarthritis Cartilage 10: 535-541.

Peterson L, Brittberg M, Kiviranta I, Akerlund EL, Lindahl A (2002) Autologous chondrocyte transplantation. Biomechanics and long-term durability. Am J Sports Med 30: 2-12.

Raum K (2008) Microelastic imaging of bone. IEEE Trans Ultrason Ferroelectr Freq Control 55: 1417-1431.

Raum K, Gottwald M, Wohlrab D, Göbel F (2007) Depth dependent high frequency backscatter analysis of degenerated cartilage. IEEE Ultrasonics Symposium Proceedings 1: 1109-1112. 
Raum K, Hofmann T, Leguerney I, Saied A, Peyrin F, Vico L, Laugier P (2007) Variations of microstructure, mineral density and tissue elasticity in $\mathrm{b} 6 / \mathrm{c} 3 \mathrm{~h}$ mice. Bone 41: 1017-1024.

Raum K, Leguerney I, Chandelier F, Talmant M, Saied A, Peyrin F, Laugier P (2006) Site-matched assessment of structural and tissue properties of cortical bone using scanning acoustic microscopy and synchrotron radiation muct. Phys Med Biol 51: 733-746.

Rengle A, Armenean M, Bolbos R, Goebel JC, Pinzano-Watrin A, Saint-Jalmes H, Gillet P, Beuf O (2009) A dedicated two-channel phased-array receiver coil for high-resolution mri of the rat knee cartilage at $7 \mathrm{~T}$. IEEE Trans Biomed Eng 56: 2891-2897.

Saarakkala S, Jurvelin JS, Zheng YP, Nieminen HJ, Toyras J (2007) Quantitative information from ultrasound evaluation of articular cartilage should be interpreted with care. Arthroscopy 23: 1137-8; author reply 1139-1141.

Saarakkala S, Laasanen MS, Jurvelin JS, Toyras J (2006) Quantitative ultrasound imaging detects degenerative changes in articular cartilage surface and subchondral bone. Phys Med Biol 51: 5333-5346.

Saris DB, Vanlauwe J, Victor J, Haspl M, Bohnsack M, Fortems Y, Vandekerckhove B, Almqvist KF, Claes T, Handelberg F, Lagae K, van der Bauwhede J, Vandenneucker $\mathrm{H}$, Yang KG, Jelic M, Verdonk R, Veulemans N, Bellemans J, Luyten FP (2008) Characterized chondrocyte implantation results in better structural repair when treating symptomatic cartilage defects of the knee in a randomized controlled trial versus microfracture. Am J Sports Med 36: 235-246.

Spriet MP, Girard CA, Foster SF, Harasiewicz K, Holdsworth DW, Laverty S (2005) Validation of a $40 \mathrm{Mhz}$ B-scan ultrasound biomicroscope for the evaluation of osteoarthritis lesions in an animal model. Osteoarthritis Cartilage 13: 171-179.
Toyras J, Laasanen MS, Saarakkala S, Lammi MJ, Rieppo J, Kurkijarvi J, Lappalainen R, Jurvelin JS (2003) Speed of sound in normal and degenerated bovine articular cartilage. Ultrasound Med Biol 29: 447-454.

Toyras J, Rieppo J, Nieminen MT, Helminen HJ, Jurvelin JS (1999) Characterization of enzymatically induced degradation of articular cartilage using high frequency ultrasound. Phys Med Biol 44: 2723-2733.

Viren T, Saarakkala S, Kaleva E, Nieminen HJ, Jurvelin JS, Toyras J (2009) Minimally invasive ultrasound method for intra-articular diagnostics of cartilage degeneration. Ultrasound Med Biol 35: 1546-1554.

Wang Q, Zheng YP, Qin L, Huang QH, Lam WL, Leung G, Guo X, Lu HB (2008) Real-time ultrasonic assessment of progressive proteoglycan depletion in articular cartilage. Ultrasound Med Biol 34: 1085-1092.

Wasiak J, Clar C, Villanueva E (2006) Autologous cartilage implantation for full thickness articular cartilage defects of the knee. Cochrane Database Syst Rev 3: CD003323.

Xu JW, Zaporojan V, Peretti GM, Roses RE, Morse KB, Roy AK, Mesa JM, Randolph MA, Bonassar LJ, Yaremchuk MJ (2004) Injectable tissue-engineered cartilage with different chondrocyte sources. Plast Reconstr Surg 113: 1361-1371.

Zheng YP, Huang YP (2008) More intrinsic parameters should be used in assessing degeneration of articular cartilage with quantitative ultrasound. Arthritis Res Ther 10: 125 .

Zizak I, Roschger P, Paris O, Misof BM, Berzlanovich A, Bernstorff S, Amenitsch H, Klaushofer K, Fratzl P (2003) Characteristics of mineral particles in the human bone/cartilage interface. J Struct Biol 141: 208-217. 